\title{
Postsurgical high grade transformation of polymorphous low grade adenocarcinoma of the retromolar trigone into adenocarcinoma not otherwise specified
}

\author{
Ranmeet Batra, Harshaminder K Grewal \\ 1. Department of Oral Surgery, Himachal Institute of Dental Sciences, Paonta Sahib (Sirmour, H.P.), India. 2. Department \\ of Oral Pathology, M.M. Institute of Dental Sciences and Research, M. M. University, Mullana, Ambala (Haryana), India.
}

Correspondence: Ranmeet Batra. Address: Waryam Singh Hospital, Jagdhri Road, Yamunanagar 135001 (Haryana), India. E-mail: ranmeetbatra@gmail.com

Received: March 4, 2014

Accepted: June 24, 2014

Online Published: June 26, 2014

DOI : $10.5430 /$ crcp.v1n2p141

URL: http://dx.doi.org/10.5430/crcp.v1n2p141

\begin{abstract}
Malignant salivary gland tumors have been known to undergo transformation into more aggressive neoplasms with poorer prognosis in long standing or recurrent cases. This may be the result of the cell of origin to differentiate to the level of the original tumor or dedifferentiation of previously existing tumor cells. Dedifferentiation of Polymorphous low grade adenocarcinomas (PLGA) or transformation into a high grade component in recurring lesions has been reported. On transformation into high grade malignancy, salivary gland tumors do not show a total loss of original line of differentiation, especially when high grade adenocarcinomas arise from pre-existing carcinomas. Thus the term "High grade transformation" is preferred to "dedifferentiation" in these neoplasms. We present a case of PLGA of the right alveolar ridge in a 50 year old male, showing high grade transformation into Adenocarcinoma not otherwise specified (ANOS), after a period of five years of complete surgical removal.
\end{abstract}

\section{Key words}

Adenocarcinoma NOS, Retromolar trigone, Minor salivary glands, High grade transformation

\section{I ntroduction}

Malignant salivary gland tumors have rarely been known to undergo transformation into more aggressive neoplasms with poorer prognosis in long standing or recurrent cases. This may be the result of dedifferentiation of previously existing tumor cells or may occur as a result of the cell of origin of the original tumor to further dedifferentiate to the level of a more aggressive neoplasm. In tumors of bone and soft tissues, the secondary tumor looses the distinct histological features of the original tumor leading to the formation of the poorly differentiated or undifferentiated neoplasm. This process has been referred to as dedifferentiation. "Dedifferentiation" is thus defined as the abrupt transformation of well differentiated tumor into High Grade morphology that lacks the original distinct histologic characteristics ${ }^{[1]}$.

Previously known as lobular carcinoma and terminal duct carcinoma ${ }^{[2,3]}$, Polymorphous low grade adenocarcinomas (PLGA) are indolent tumours affecting the minor salivary glands almost exclusively ${ }^{[4]}$ with a preponderance for occurring in the sixth to seventh decade of life. In spite of its locally infiltrative growth pattern and perineural and perivascular invasive characteristics, PLGA is generally known to be clinically innocuous with a low grade malignant potential. Distant Published by Sciedu Press 
metastasis have been is rare, occurring in less than $1 \%$ of cases ${ }^{[5]}$. Local recurrence of PLGA after 2-14 years is not unknown. Also few reports have shown the transformation of PLGA into a more aggressive or high grade malignancy ${ }^{[1]}$.

We present a case of PLGA of the right alveolar ridge in a 50 year old male, showing high grade transformation into Adenocarcinoma not otherwise specified (ANOS) after a period of five years of complete surgical removal.

\section{Case presentation}

A 50 year old male patient presented with a painless slowly growing lesion of two years duration in the left retromolar region. Clinical oral examination revealed a sessile growth of approximately $3 \mathrm{~cm}$ diameter in the left mandibular region in relation to permanent mandibular 2nd molar. The growth showed no change in color from the surrounding mucosa, clinically resembling a peripheral giant cell granuloma or a peripheral ossifying fibroma.

Excisional biopsy revealed the presence of round cells with amphophilic cytoplasm arranged in solid, papillary-cystic and lobular patterns surrounded by scanty to moderate connective tissue stroma suggesting the presence of PLGA (see Figure 1). Immunohistochemically, both the luminal and non luminal cells showed positivity for EMA and were negative for CD43. Ki 67 showed only mild reactivity in all the tumor cells (see Figure 2). These findings further assisted in the diagnosis of PLGA. Following excision, the patient was asymptomatic for the next five years after which he complained of pain in the the same region. Intraoral examination revealed an irregular diffuse erythematous swelling measuring $3 \mathrm{~cm} \times 4$ cm (see Figure 3) extending upto the retromolar trigone. Intraoral periapical radiograph (IOPA) ruled out any bony involvement. The left submandibular lymph nodes were palpable, non-tender and not fixed to the underlying structures. Histopathological examination revealed the presence of cells arranged in duct like pattern, showing variable degree of atypia (see Figure 4). Malignant cells showed bland pale staining nucleus with little cytoplasm. In some areas, these cells showed hyperchromasia and the presence of mitotic figures (see Figure 5). The tumor cells were arranged in an organoid pattern with well defined basement membrane. Numerous clear cells (PAS negative and Mucicarmine negative) were also evident with few having centrally placed nucleus and others giving a signet ring appearance. Pools of mucoid material were also seen among the tumor cells. Minimal necrosis, restricted to necrosis of individual cells, was seen. Histopathological features suggested the presence of ANOS. The presence of ANOS at the site of a previously diagnosed and treated adenocarcinoma suggested the recurrence of the tumor with a higher grade of malignancy than the primary lesion. The quality and quantity of glandular formation was 50\% with moderate degree of nuclear atypia. Mitotic activity was seen with an average of four mitotic figures per 10 high power fields. All these features categorized the present tumor into moderately differentiated ANOS according to the histopathologic grading criteria given by Mastuba et al. ${ }^{[6]}$. The presence of mucin pools further subclassified the neoplasm as its mucinous variant. Further, when the lesion was assessed immunohistochemically using EMA, CD 43 and Ki 67, the cells showed moderate to strong positivity for EMA, focal positivity with CD43 and an intense positivity for Ki-67 (see Figure 6). Treatment included complete excision of the lesion with supraomohyoid neck dissection.

Figure 1. Low power photomicrograph (This figure shows the presence of round cells with amphophilic cytoplasm arranged in solid, papillary-cystic and lobular patterns surrounded by scanty to moderate connective tissue stroma).

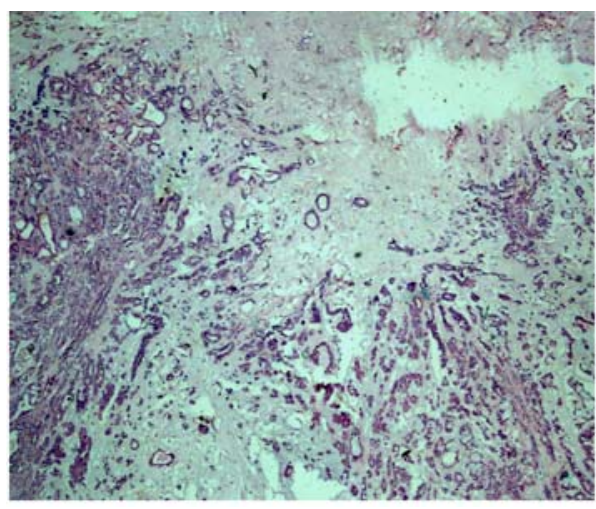


Figure 2. Photomicrograph (This figure of the primary lesion shows mild immunohistochemical staining with Epithelial Membrane Antigen [EMA] and negative staining with Ki 67).

Figure 3. Intra oral photograph (This figure shows the presence of an irregular diffuse erythematous swelling measuring $3 \mathrm{~cm} \times 4 \mathrm{~cm}$ located in the left retromolar area).

Figure 4. Low power photomicrograph (This figure shows the presence of the presence of cells arranged in duct like pattern, showing variable degree of atypia. Malignant cells showed bland pale staining nucleus with little cytoplasm. Areas of hemorrhage and necrosis are also evident).

Figure 5. High power (40×) photomicrograph (This figure shows tumor cells arranged in an organoid pattern with well defined basement membrane. Cells with hyperchromasia and the presence of mitotic figures are also evident).

Figure 6. Photomicrograph (This figure of the recurrent lesion shows intense to moderate immunohistochemical staining with Epithelial Membrane Antigen [EMA] and Ki 67).
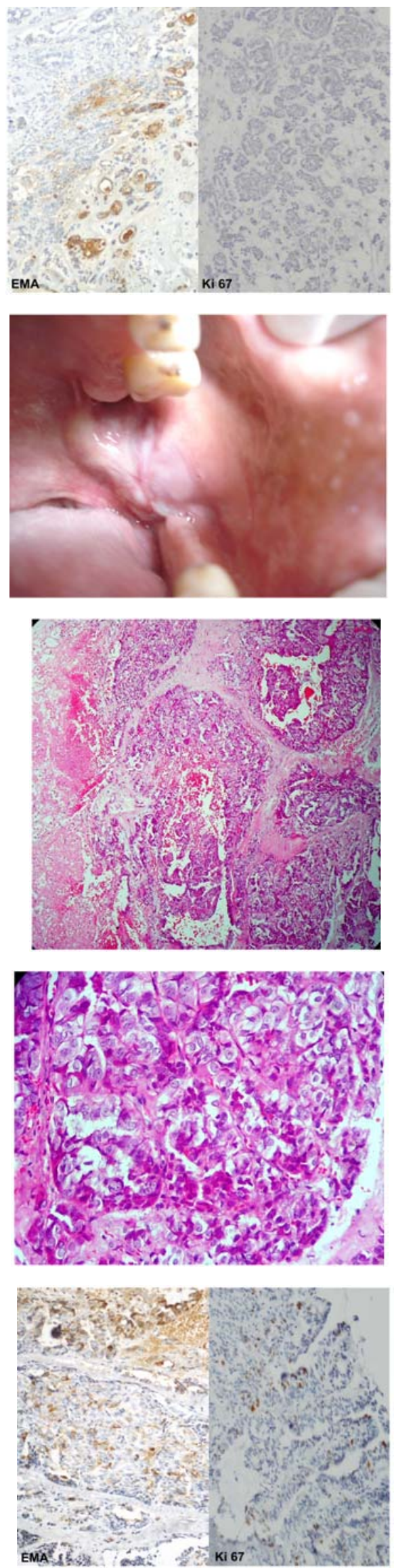


\section{Discussion}

PLGA is a distinctive salivary gland neoplasm with an almost exclusive propensity to arise from minor salivary glands. It has a predilection for females with female:male ratio of 2:1. The age ranges from 23 years to 94 years, with an average of 57.6 years at the time of presentation. In a study done in 1994, 164 cases of PLGA diagnosed between 1970-1994 were retrieved from the files of the Armed Forces Institute of Pathology of Washington DC. In this study 143 patients (87\%) presented clinically with a mass lesion, 17 of whom described a slowly enlarging mass and another 13 of whom presented with a mass accompanied by pain ${ }^{[7]}$. the initial lesion in the present case manifested as a slowly enlarging painless mass. The present case of PLGA was predominantly a papillary variant. Vincent et al. (1994) suggested that PLGA with predominant papillary configuration has a stronger tendency to recurr and metastasize ${ }^{[8]}$. Dedifferentiation of PLGA or transformation into a high grade component in recurring lesions have been reported. Simpson RH et al. in 2002 reported two cases of PLGA showing high grade transformation with one case showing transformation into high grade carcinoma with prominent zones of necrosis and MIB 1 index and another case with a high grade transformation after 13 years of initial biopsy ${ }^{[5]}$. On transformation into a higher grade malignancy, salivary gland tumors do not show a total loss of original line of differentiation, especially when high grade adenocarcinomas arise from pre-existing carcinomas. Thus, the term "high grade transformation" is preferred to "dedifferentiation" in these neoplasms ${ }^{[1]}$.

According to Batsakis et al. (1992), PLGA originates from intercalated ducts ${ }^{[8]}$ and Adenocarcinoma NOS has been hypothesized to arise from the ductal reserve cell ${ }^{[9]}$. But in the present case, ANOS may have occurred as a recurring more aggressive lesion after the complete surgical excision of pre-existing PLGA. Mills SE et al. in 1984 reported a case of PLGA that underwent transformation into solid adenocarcinoma, eventually leading to death of the patient ${ }^{[10]}$. Also, Pelkey TJ and Mills SE in 1999 reported 2 cases of PLGA arising in the palate and associated with multiple locoregional recurrences, treated with excision and radiation therapy that showed high grade transformation after 17 and 26 years, respectively ${ }^{[11]}$ and, Thennavan A et al. in 2013 reported a 73-year-old female patient with PLGA of the maxillary alveolus which exhibited high-grade transformation and subsequently metastasis to the abdomen and lungs ${ }^{[12]}$.

During tumorigenesis, the ductal reserve cells progress to bi-multi diferentiation. This explains the architecture of ANOS which consists predominantly of glandular or solid structures accompanied by papillar cystic or salivary duct carcinoma like pattern. The presence of necrosis in the recurrent lesion, the increase in the immunohistochemical expression of EMA and $\mathrm{Ki}-67$, and the change in the expression of CD 43 from negative in the primary tumor to positive in the recurrent tumor suggests transformation of a low grade PLGA into ANOS, which is a high grade malignancy compared to PLGA.

\section{Clinical significance}

Spiro and collegues in their study of 204 patients found cervical metastasis in $27 \%$ cases out of which more than half showed cervical metastasis at the initial time of diagnosis ${ }^{[13]}$. Matsuba and colleagues have recorded a median survival of four years for all 54 patients with ANOS in their study group (including Sino nasal sites) ${ }^{[6]}$. Cervical lymph node metastases developed in 23\% of patients, and $73 \%$ died within three years. Distant metastases developed in 37\% of patients, and $93 \%$ died within three years. Distant metastasis to the lungs is seen in $26 \%$ cases. ANOS rarely metastasizes to the skeleton, abdomen and skin. Wahlberg et al. ${ }^{[14]}$ reviewed National Swedish Cancer Registry data on 2,465 patients and found a significantly decreased overall survival for patients with ANOS as compared to those with other more common salivary malignancies. According to them, the overall prognosis of adenocarcinoma NOS depended on clinical stage and histopathological grade ${ }^{[15]}$. According to Spiro et al. the five year cure rates for grade I, II and III ANOS have been reported to be $69 \%, 46 \%$ and $8 \%$ respectively and those for 15 years have been reported as $54 \%$, 31\% and $3 \%$ respectively ${ }^{[13]}$. Clinical stage, particularly tumor size may be the critical factor to determine the outcome of salivary gland cancer and may be more important than histologic grade in this respect ${ }^{[16]}$. In a 15 year followup by Spiro et al. they found cure rate for clinical stages I, II and III of ANOS to be $67 \%$, 35\% and $8 \%$ respectively ${ }^{[13]}$. Therefore clinical staging was done in the present case, where, the size of swelling was $2 \mathrm{~cm} \times 1.5 \mathrm{~cm}$, with metastasis seen in a single ipsilateral lymph node, $<3 \mathrm{~cm}$ in dimension, thus being categorized under stage III according to TNM staging system. Five year followup of the present case showed the patient free of any recurrence. 


\section{Conclusion}

Though PLGA is considered as a low grade salivary gland malignancy with infrequent recurrence and metastasis specially when occuring in minor salivary glands, it may undergo high grade transformation and recurr as high grade malignancies. Thus, carefully excising the neoplasm with adequate margins and long term followups should be mandatory.

\section{References}

[1] Toshitaka Nagao. "Dedifferentiation” and High-Grade Transformation in Salivary Gland Carcinomas Head and Neck Pathol. 2013; 7: S37-S47.

[2] Freedman PD, Lumerman H. Lobular carcinoma of intraoral minor salivary gland origin. Report of twelve cases. Oral Surg Oral Med Oral Pathol. 1983; 56: 157-66. http://dx.doi.org/10.1016/0030-4220(83)90282-7

[3] Anderson C, Krutchkoff D, Pedersen C, Cartun R, Berman M. Polymorphous low grade adenocarcinoma of minor salivary gland: a clinicopathologic and comparative immunohistochemical study. Mod Pathol. 1990; 3: 76-82. PMid: 2308924.

[4] Hannen EJ, Bulten J, Festen J, et al. Polymorphous lowgrade adenocarcinoma with distant metastases and deletions on chromosome 6q23-qter and 11q23-qter: a case report. J Clin Pathol. 2000; 53: 942-945. PMid: 11265182.

http://dx.doi.org/10.1136/jcp.53.12.942

[5] Simpson RH, Pereira EM, Ribeiro AC, et al. Polymorphous low grade adenocarcinoma of the salivary glands with transformation to high-grade carcinoma. Histopathology. 2002; 41: 250-259. PMid: 12207787.

http://dx.doi.org/10.1046/j.1365-2559.2002.01439.x

[6] Mastuba HM, Mauncy M, Simpson JR, Thawley SE, Pikul FJ. Adenocarcinomas of major and minor salivary gland origin: A histopathologic review of treatment failure patterns. Laryngoscope. 1988; 98: 784-788.

[7] James T. Castle JT, Thompson Lester DR, Frommelt RA, Wenig BM, Kessler HP. Polymorphous Low Grade Adenocarcinoma: A Clinicopathologic Study of 164 Cases. CANCER. 1999; 86(2): 207-19. http://dx.doi.org/10.1002/(SICI)1097-0142(19990715)86:2<207::AID-CNCR4>3.0.CO;2-Q

[8] Cawson RA, Gleeson MJ, Eveson JW. Pathology and surgery of the salivary glands. Publisher ISIS Medical Media, Oxford. Editor Cawson RA (Roderick). Published in 1997. 1st ed.

[9] Dwivedi N, Aggarwal A, Raj V, Chandra S. Histogenesis of salivary gland neoplasms. Indian J Cancer. 2013; 50: 361-6. Mid: 24369218. http://dx.doi.org/10.4103/0019-509X.123629

[10] Mills SE, Garland TA, Allen MS Jr. Low-grade papillary adenocarcinoma of palatal salivary gland origin. Am J Surg Pathol. 1984 May; 8(5): 367-74. PMid: 6731663. http://dx.doi.org/10.1097/00000478-198405000-00005

[11] Pelkey TJ, Mills SE. Histologic transformation of polymorphous low-grade adenocarcinoma of salivary gland. Am J Clin Pathol. 1999 Jun; 111(6): 785-91. PMid: 10361514.

[12] Aatish Thennavan, Lakshmi Rao, Raghu Radhakrishnan. Polymorphous low-grade adenocarcinoma of maxillary alveolus metastasising to the abdomen: the role of immunomarkers in diagnosis. BMJ Case Reports. 2013. http://dx.doi.org/10.1136/bcr-2013-009633

[13] Spiro RH. Factors affecting survival in salivary gland cancers. In: McGurk M, Renehan AG, eds. Controversies in the Management of Salivary gland disease, Oxford, UK: Oxford University Press. 2001; 143-150.

[14] Wahlberg P, Anderson H, Biorklund A, et al. Carcinoma of the parotid and submandibular glands: a study of survival in 2465 patients. Oral Oncol. 2002; 38: 706-713. http://dx.doi.org/10.1016/S1368-8375(02)00007-6

[15] Auclair PL, Ellis GL. Adenocarcinoma Not Otherwise Specified. In: Mitchell J ed. Surgical pathology of the salivary glands. Philadelphia; W.B. Saunders. 1991: 318-332. (Major problems in pathology; vol 25)

[16] Edge SB, Byrd DR, Campton CC, et al. Major salivary glands (parotid, submandibular and sublingual). In: AJCC Cancer Staging Manual. 7th ed. New York (NY): Springer. 2010; 79-82. 